\title{
Factor XII inhibition is neuroprotective after traumatic brain injury in mice
}

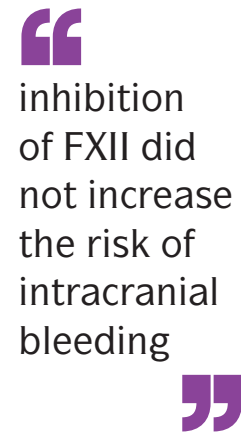

Inhibition of coagulation factor XII (FXII) activity after traumatic brain injury (TBI) could minimize secondary brain damage without the risk of inducing bleeding, according to a new mouse study. Thrombosis formation in two mouse models of TBI was reduced in the absence of FXII activity; translation of the findings to humans could provide the first safe TBI intervention.

Microvascular thrombosis contributes to secondary brain damage after TBI by causing ischaemic injury, but the use of anticoagulants to prevent thrombosis is controversial, as it is associated with an increased risk of intracerebral haemorrhage. However, previous work has identified FXII

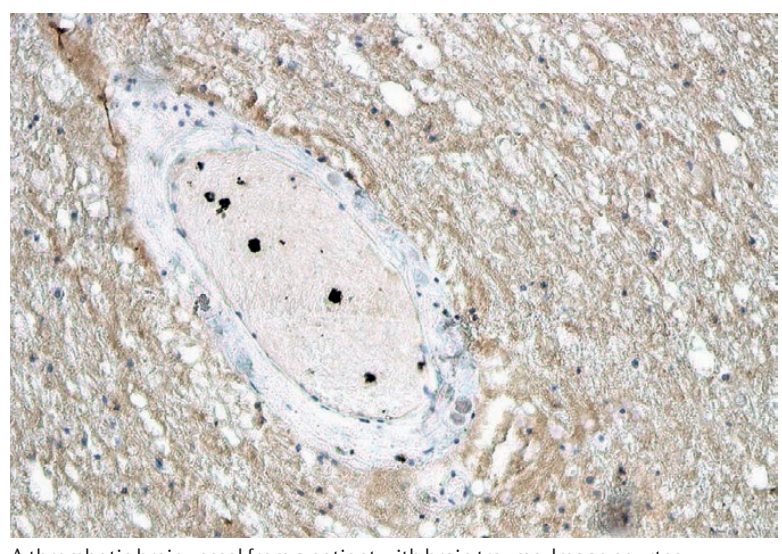

A thrombotic brain vessel from a patient with brain trauma. Image courtesy of C. Kleinschnitz. as a component of the clotting pathway that has unique potential to overcome this problem.

"Deficiency of FXII in patients is not associated with a bleeding phenotype, but FXII-deficient mice have severely impaired thrombus formation," explains Christoph Kleinschnitz, who led the new study. "We previously showed that genetic deletion or pharmacological inhibition of FXII reduced ischaemic lesions in mouse models of ischaemic stroke without increasing intracranial haemorrhages."

In their new study, Kleinschnitz and colleagues aimed to determine whether a similar effect could be seen in two mouse models of TBI. They used a weight-drop injury model, which induced diffuse brain trauma, and a cortical cryolesion model of focal lesions. In both models, injury triggered microvascular occlusion and accumulation of platelets, pathology that mirrored findings in 10 brain tissue samples from humans with TBI.

In both models, genetic deletion of FXII reduced the amount of vessel occlusion and the number of platelets in the brain tissue. Intravenous injection of human FXII into knockout mice, however, negated the effect of the deletion, demonstrating that FXII is sufficient to induce thrombosis after TBI regardless of the nature of the initial injury. These effects translated into improved outcomes after TBI, including better motor function, reduced brain lesion volumes and less neurodegeneration.

Kleinschnitz and co-workers also took the first step toward exploiting the effects of FXII on thrombosis in the management of TBI. They tested pharmacological inhibition of FXII after TBI in mice by injecting the animals with recombinant human albumin-fused infestin 4 . The resulting inhibition produced the same effects as genetic deletion of FXII: less thrombosis, lower lesion volumes and improved outcomes after TBI.

Importantly, inhibition of FXII did not increase the risk of intracranial bleeding in the mice, meaning that it could be a safer alternative to traditional anticoagulants for preventing thrombosis. "Our findings show that targeting of activated FXII with compounds such as infestin 4 might provide a novel treatment option for TBI," concludes Kleinschnitz.

Ian Fyfe

ORIGINAL ARTICLE Hopp, S. et al. Targeting coagulation factor XII as a novel therapeutic option in brain trauma. Ann. Neurol. http://dx.doi. org/10.1002/ana.24655 (2016) 